\title{
A Novel Allocation Algorithm for Evacuation Simulation Based on Cellular Automaton
}

\author{
Fei Fang and WenchunYu \\ Engineering and Technology College of NeiJiang Normal University, NeiJiang \\ Sichuan 641110, China \\ fangfei_nj@163.com; ywclmxx@163.com
}

\begin{abstract}
In this paper we study the evacuation simulation in buildings using the cellular automaton theory. First, we study the rules of path selection in personnel evacuation. Second, we introduce the individual competence to solve the conflict problem caused by multiple people compete for the same cell. Third, we propose a novel evacuation allocation algorithm and improved the simulation model. The proposed model takes the individual competence, the conformist mentality, and the fog influence into consideration. The experimental results show that the improved simulation model is reliable and close to the actual situation, and we hope this work may contribute to the design of evacuation systems in buildings.
\end{abstract}

Keywords: Personnel evacuation; Cellular automaton; Individual competence; Allocation algorithm; Simulation model

\section{Introduction}

Personnel evacuation is an important and hot research area. With the development of computer technology, the computer simulation has become the major approach to study personnel evacuation. The current evacuation model includes two types, the micro model and the macro model. The macro model mainly concerns the evacuation ability of the building, and overlooked the individual variance. The micro model [1-3] considers the individual movement in discrete forms, and decreases the simulation computing cost. When the simulation model is suitable, the result will be accurate. Using the micro model, we can analyze the detailed personnel behavior feature in the evacuation. It has drawn more and more research attentions recently. Some researchers have studied the exit in evacuation, as well as the closed space problem[4]. Most of the simulation model have not consider the individual variance and the confliction problem caused by multiple individuals compete for the same spot. In this paper, we consider the individual competence, and introduce the conflict detection and allocation algorithm. Using cellular automaton, which is a micro simulation model, we study the evacuation model in buildings.

Cellular Automaton (pl. Cellular Automata, CA) is defined on the cellular space with discrete and finite-state cells. Following certain local rules, it is a dynamic system evolving on discrete time dimension. Each cell, distributed in the grid of rules, takes finite discrete states. It is updated based on the fixed local rules. A large number of cells may become a dynamic evolving system though simple connection.

\section{Personnel Evacuation Path Selection Rule}

In the cellular automaton model, the evacuation area in fire is separated into grids using identical rectangles. Each grid is considered as a cell, and the cellular space is consisted of all cells. In our model, only one person can be fit in one cell, e.g. $0.4 \mathrm{~m} \times 0.4 \mathrm{~m}$. This is a 
typical space allocation of dense crowd. Within any given time step, a cell my take one of several states ( in binary condition, 0 or 1 ). Each cell state is determined by its previous state and the neighboring cell states.

In the evacuation, the cell with the maximum attraction among the current cell state and the neighboring cell states is chosen as the target cell in the next time[5-6]. When we calculate the probability of certain candidate cell, we consider the distance from the target cell to the exit, the conformist mentality, the familiarity of the environment, and the fog influences. First, we use the exit attraction [7-8] and the environment attraction [9-10] to calculate the probability, the other factors will be considered later:

$$
p_{1}=k_{1} p_{\text {ent }(i, j)}+k_{2} p_{\text {aru }(i, j)}
$$

In Equation (1), $p_{1}$ is the probability of the candidate cell, $p_{\text {ent }(i, j)}$ is the probability of the exit attraction, $p_{a r u(i, j)}$ is the probability of the environment attraction, $k_{1}$ is the environment familiarity level, and $k_{2}$ is the factor of conformist mentality

$$
p_{\text {ent }}=\frac{d_{\max }-d_{i j}}{d_{\max }}
$$

The exit attraction calculation is given in Equation (2). When the current cell is the exit, the attraction equals to1.

$$
P_{\text {aru }(i, j)}=\frac{\operatorname{count}(i, j)}{\operatorname{sum}(i, j)}
$$

The environment attraction calculation is given in Equation (3). The conformist mentality in emergency is considered, the neighboring cell with a higher summed chosen times by other people is preferred coun(i, $j)$ denotes how many times the current cell $(i, j)$ has been chosen, $\operatorname{sum}(i, j)$ denotes the total summed times of the neighboring 9 cells.

$$
p=p_{1} \times s
$$

In Equation (4), $P$ is the final candidate cell probability, $p_{1}$ is the probability given by Equation (1), $s$ is the decreasing factor caused by fog and $s$ is between 0 to 1 .

\section{Individual Competence and Conflict Detection}

\subsection{Individual Competence}

In evacuation simulation, the same target cell may be chosen as the optimized target by multiple people. We use competence[11] to solve this problem. The individual competence is based on the person's physical condition and the strength condition. When the strength condition is strong, the competence is high. When the person is hurt by fog or high temperature, the competence drops. When death happens, the competence is set to 0 .

$$
E=B^{*} C
$$

In Equation (5), $\mathrm{E}$ denotes the competence, $\mathrm{B}$ is the physical condition, $\mathrm{C}$ is the strength condition.

$$
B=H^{*} S^{*} Y
$$

In Equation (6), we calculate the physical condition, where $\mathrm{H}$ is the height, $S$ is the gender, and $Y$ is the age. $H$ may takes the following values in Equation (7).

$$
H=\left\{\begin{array}{cc}
1 & H_{m}>=1.8 \| H_{w}>=1.7 \\
0.85 & H_{m}>=1.75 \| H_{w}>=1.65 \\
0.8 & H_{m}>=1.7 \| H_{w}>=1.6 \\
0.75 & H_{m}>=1.6 \| H_{w}>=1.5 \\
0.7 & H_{m}<1.6 \| H_{w}<1.5
\end{array}\right.
$$


In Equation (7), $H_{w}$ denotes the height of a male, and $H_{m}$ denotes the height of a female. The male is given the factor $S=1$, and for female, $S=0.9$.

$$
Y=\sin \left(\pi * \frac{70-a}{70}\right)
$$

In Equation (8), the age factor is given, where a is the actual age. When it is bigger than 70 , let $\mathrm{a}=70, \mathrm{C}$ takes a random number between 0 to 10 . As the evacuation goes on, the strength condition factor drops. When the person is exposed to thick fog or close to fire source, the strength will drop very fast, until 0 , which means death.

\subsection{Conflict Detection}

When a fire happens, or in other similar emergency, individual will take action to protect himself at the same time. In the evacuation simulation model, we need to consider this parallel behaviors. When the confliction is caused by such behaviors, we need a good allocation algorithm to solve this essential problem in evacuation model. In Figure 1, we demonstrated the situation in which 8 people is competing for the same exit.

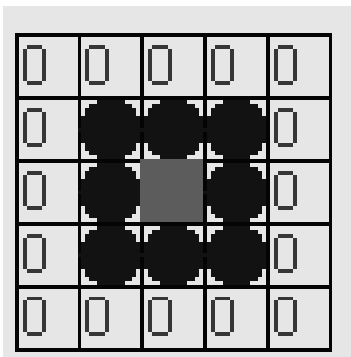

\section{Figure 1. Multiple People Compete For the Same Exit}

In the current evacuation model, two type of solutions [13] may be used to solve the confliction problem. In the first type of solution, at the beginning of each time step, we randomly decide the priority of each people. And then we calculate the probability and move people according to this order. We will move the people of high priority first to the target cell, and move the low priority people later. In the second type of solution, we allow all the individuals to make the decision at the same time, and then use certain rules to eliminate the conflict.

The first type of solution can avoid the confliction and save computation cost, therefore it is easy to implement. Most of the current model use this type of solution. However, it is not close to the real world situating. In the actual evacuation, each individual takes action at the same time, not one by one. The individual competence needs to be considered. When the same cell is chosen as the target cell by multiple people, we need certain rule to solve the conflict.

\section{Optimization Algorithm}

In order to better simulate the parallel behavior character of personnel evacuation, we use the second type of solution to solve conflict. We propose a conflict detection and removing algorithm. Detailed algorithm is given below:

(1) At the beginning of each time step, we first calculate the candidate cell for each person. A candidate list is created to store the candidate cell for each person, including the current cell. The cells will be ranked according to the attraction, from the biggest to the smallest. The biggest cell is the first candidate in the list.

(2) Create a list of conflict. Each element in the list is a predefined structure. The structure is consisted of two parts, a cell ID and a reference list. The cell ID $a$ stored here 
is the first candidate in each person's list. When multiple people choose the same cell, we can see the same cell ID $a$ in multiple element in the conflict list. The reference list is used to store all the people who choose the same cell ID $a$. We name this type of data structure as first candidate personnel list. Before conflict, it is empty, nobody has chosen the same cell yet.

(3) In conflict, we travel through each person, when the current person is $i$, first check if there exist a conflict list element in which the cell ID is the same as the first candidate of person $i$ 's list. If found the same ID, we add this person to the reference list of the same element in the conflict list. If the same cell ID is not found in the conflict list, then we check the rest of the persons from $i+1$. If we found anyone whose list has the same candidate cell as the current person $i$, we create an element in the conflict list using the current person's candidate cell ID. Go to the beginning and process the next person $i+1$ until we travelled through everyone.

(4) Check the conflict list, if the list is empty, end the process. If it is not empty, we calculate the competence of each person in the reference list with the same cell ID. Rank the competences and find the person with highest competence. We then keep the candidate cell in this person's list, and delete the first candidate in all other person's candidate list. The second candidate becomes the first candidate. Delete the conflict list and go to Step (2) to continue conflict detection.

The main title (on the first page) should begin $13 / 16$ inches ( 7 picas) from the top edge of the page, centered, and in Times New Roman 14-point, boldface type. Capitalize the first letter of nouns, pronouns, verbs, adjectives, and adverbs; do not capitalize articles, coordinate conjunctions, or prepositions (unless the title begins with such a word). Please initially capitalize only the first word in other titles, including section titles and first, second, and third-order headings (for example, "Titles and headings" — as in these guidelines). Leave two blank lines after the title.

\section{Simulation and Analysis}

\subsection{The Influence of Individual Competence}

In this section we set up an experiment to analyze the influence of individual competence on the evacuation process. In Figure 2, the properties of person A and B are given in Table 1. The competence is calculated by the equations given in Section 2. In Equation (1), $k_{1}$ is set to 0.5 .

Table 1. Properties of Person A and B in Evacuation

\begin{tabular}{ccc}
\hline People ID & A & B \\
\hline Cell Location & $(3,2)$ & $(5,2)$ \\
Age & 12 & 35 \\
Height & $1.6 \mathrm{~m}$ & $1.75 \mathrm{~m}$ \\
Gender & Female & Male \\
Current Strength & 0.8 & 0.6 \\
E(Competence) & 0.306 & 0.51 \\
\hline
\end{tabular}




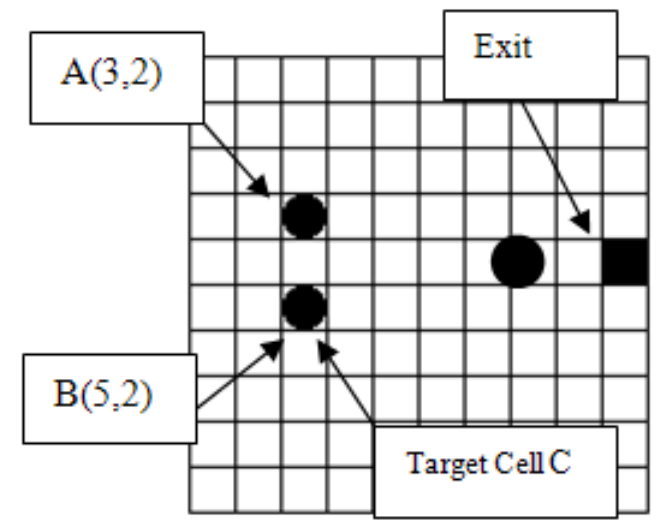

(a) Cell Location of A and B when $\mathrm{t}=0$

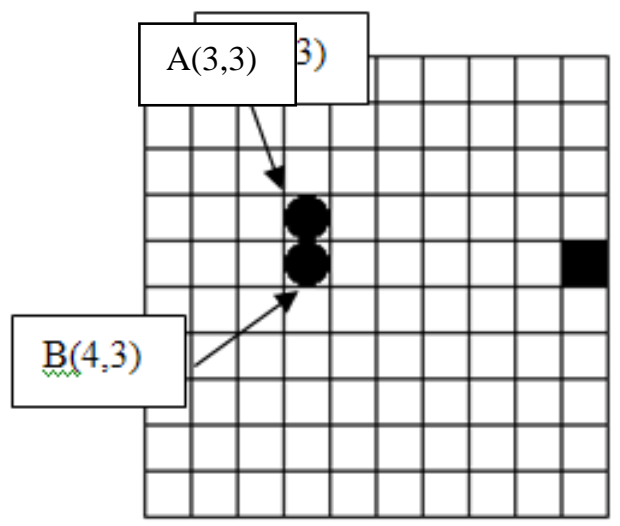

(b) Cell Location of A and B when $\mathrm{t}=1$

\section{Figure 2. Person A and B compete for Target Cell $C$ at the same time}

We can see from Figure 2 that the competence of $\mathrm{A}$ is 0.306 and the competence of $\mathrm{B}$ is 0.51 when they compete for the same target cell C. In this situation, B will get the cell $\mathrm{C}$, and A has to choose the other candidate cell with high probability in his candidate list.

\subsection{Conformist Mentality Analysis}

In the evacuation process, due to the individual variance, the physical condition, the strength condition, the familiarity of environment, and the mental state will cause different behavior. The conformist mentality is an important factor we set up different values in this experiment to analyze the time steps needed for evacuation.

We set the space as $30 \times 30$ grid, person ratio is $20 \%$, as shown in Figure 3.In Figure 3 we can see that the factor is set to $0,0.5,0.7,0.8$ and 0.95 respectively. The simulation results under five situations are show in Table 2 and Figure 4.

In Figure 2 and Table 4, we can see when the factor of conformist mentality increases, the simulation time also increases. The experimental results show that if people are familiar with the environment, they can find the exit faster and avoid following people blindly. Therefore in public buildings, we need to give a detailed evacuation map and point out the right path to the exit. When emergency happens, people may evacuate fast and avoid panic and injuries.

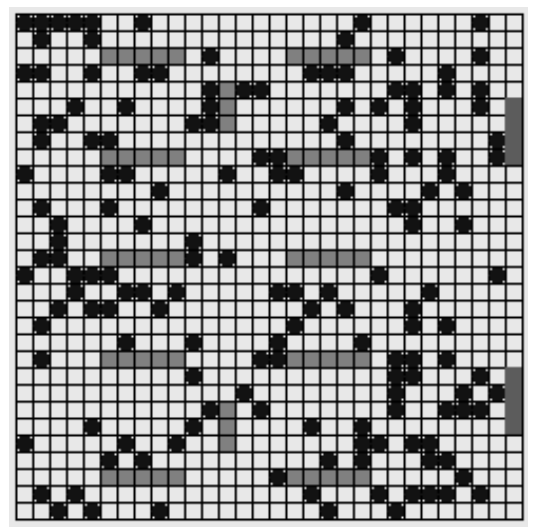

Figure 3. Conformist Mentality Experiment 
Table 2. Evacuation Time Steps under Different Factors of Conformist Mentality

\begin{tabular}{cc}
\hline Factors of Conformist Mentality & Evacuation Time Steps \\
\hline 0.0 & 40 \\
0.5 & 42 \\
0.7 & 44 \\
0.8 & 104 \\
0.9 & 126 \\
\hline
\end{tabular}

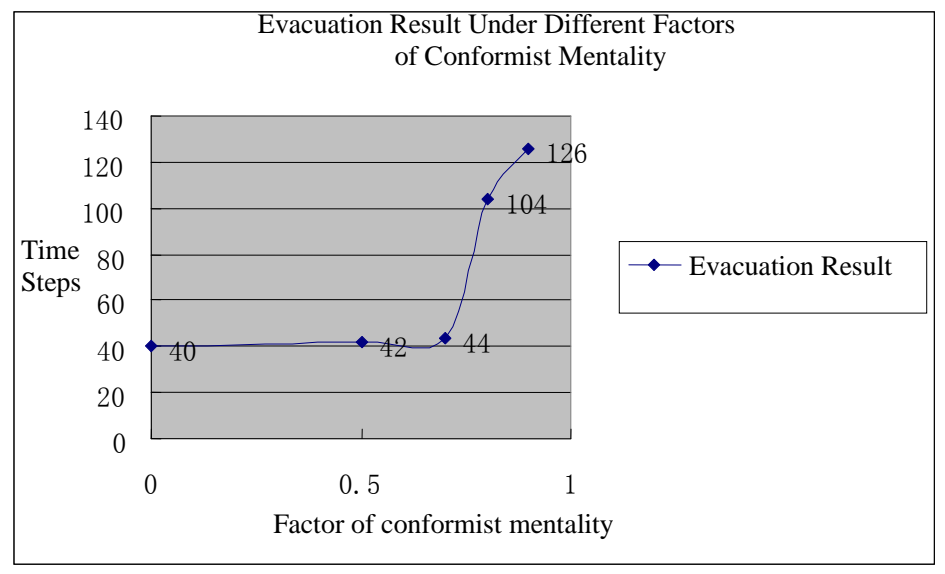

\section{Figure 4. Evacuation Result under Different Factors of Conformist Mentality}

\subsection{Fog Diffusion Analysis}

In the fire evacuation, fog is an important factor. Most of the time, people die from the poison gas or high temperature. By introducing the fog model into the simulation, we may analyze its influence on personnel evacuation.

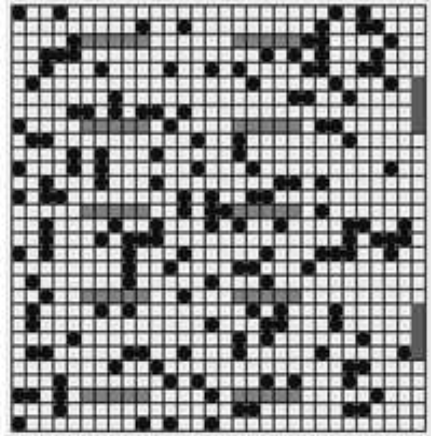

(a)

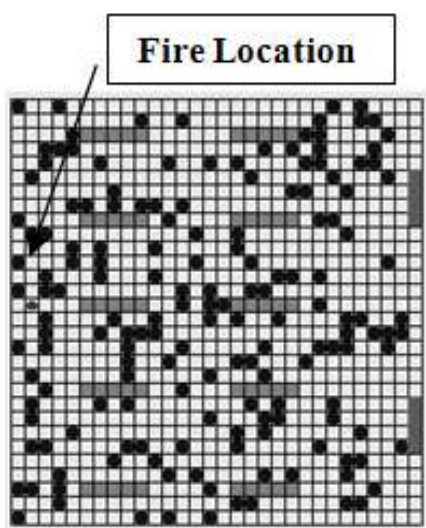

(b)

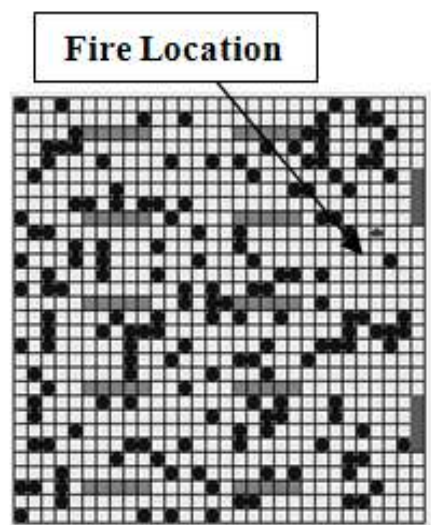

(c)

Figure 5. Fog Model Simulation

As shown in Figure 5, we create 3 experiments. In each experiment, the number of cells, the person ratio, and the individual characters are the same. The only different factor is the location of fire. In (a), there is no fire, thus no frog; in (b), the 
fire location is far away from the exit; in (c) the fire location is close to the exit. The simulation results are shown in Table 3.

\section{Table 3. Simulation Data of Fog Model}

\begin{tabular}{ccccccc}
\hline \multirow{2}{*}{$\begin{array}{c}\text { Current } \\
\text { time step }\end{array}$} & \multicolumn{2}{c}{ No fog } & \multicolumn{2}{c}{ Fire far from exit } & \multicolumn{2}{c}{ Fire close to exit } \\
\cline { 2 - 7 } & Survivals & Victims & Survivals & Victims & Survivals & Victims \\
\hline 0 & 164 & 0 & 164 & 0 & 164 & 0 \\
10 & 130 & 0 & 130 & 0 & 130 & 0 \\
20 & 80 & 0 & 80 & 0 & 80 & 0 \\
30 & 34 & 0 & 31 & 0 & 42 & 10 \\
40 & 1 & 0 & 2 & 0 & 11 & 18 \\
42 & 0 & 0 & 0 & 0 & 9 & 18 \\
50 & $/$ & $/$ & $/$ & $/$ & 2 & 20 \\
60 & $/$ & $/$ & $/$ & $/$ & 1 & 21 \\
106 & $/$ & $/$ & $/$ & $/$ & 0 & 22 \\
\hline
\end{tabular}

Table 3 shows that, when we introduce the fog factor, the simulation will be influenced significantly. The evacuation is satisfactory when the fire location is far away from the exit. However, when the fire location is close to the exit, the efficiency of the evacuation drops, and causing more personnel injuries. Therefore, in public buildings the combustibles are not allowed to put close to the exit.

\section{Conclusions}

In this paper we use cellular automaton theory to improve the evacuation simulation model. We introduce the individual competence factor to solve the conflict problem caused by multiple people compete for the same cell, and proposed a novel parallel allocation algorithm in evacuation. The experimental results show that the improved model may better simulate the actual evacuation process, and the path selected is better.

Due to the complexity in evacuation simulation, the simulation of individual' $\mathrm{s}$ physical condition and mentality character is still limited. In the future work, we may combine the cellular automaton and the intelligent agent, and bring the simulation closer to the actual evacuation problem.

\section{Acknowledgments}

This work was supported by the Sichuan Education Department under Grant No.13ZA0005.

\section{References}

[1] T. Bu and D. Towsley, "On distinguishing between Internet power law topology generators", Kermani P. Proc of INFOCOM. New York, USA: IEEE Computer Society Press, (2002), pp. 638-647.

[2] M. Faloutsos, P. Faloutsos and C. Faloutsos, "On power-law relationships of the Internet topology", Chapin L,Sterbenz J P G,ParulkarG, et al.Proc of SIGCOMM. Cambridge, Massachusetts, USA: ACM Press, (1999), pp. 251-262.

[3] M. E. J. Newman, "The structure and function of complex networks",SIAM Review, vol. 45, no. 2, (2003), pp. 167-256.

[4] F. Li, W. Fang and Q. Hu, "Simulation and analysis of passenger's evacuation ability in subway under urgent conditions", Journal of System Simulation, vol. 18, no. 4, (2006), pp. 852-855.

[5] S. Zhou, "Implementation of Occupant Evacuation Simulation System in Large Buildings", Computer Simulation, vol. 26, no. 6, (2009), pp. 191-194

[6] S. Andreas, "Cellular Automaton Approach to Pedestrian Dynamics-Theory", in: Pedestrian and Evacuation Dynamics (PED), eds .M .Schreckenberg, S .D .Sharma, Springer, Berlin, (2001).

[7] G. Jiang, "Influences of Building Exit Conditions on Pedestrian Evacuation", Computer Simulation, vol. 27 , no. 6 , (2010), pp. 212-215 
[8] P. Rao, X. Zhou, K. Zhu and L. Yang, "Cellular Automation Simulation of Pedestrian Evacuation under Complex Building Structure", China Safety Science Journal, vol. 21, no. 12, (2011), pp. 34-39

[9] Z. Lu and W. Qin, "Simulation of crowd evacuation in fire based on agent behavior", Journal of Southeast University, vol. 41, no. 6, (2011), pp. 1295-1299.

[10] X. Hu, Q. Tian, Y. Chen and X. Li, "Staff evacuation simulation of different staff distribution in highspeed rail compartment", Journal of Computer Applications, vol. 33, no. 1, (2013), pp. 281-284.

[11] Y. Zhao, M. Huang and H. Zhang, "On multi-exit occupant evacuation model based on cellular automaton", Journal of Systems Engineering, vol. 27, no. 4, (2012), pp. 439-442.

[12] P. Zhang and B. Chen, "Behavior Rules of Human Evacuation in Fire", Journal of Northeastern University, vol. 22, no. 1, (2001), pp. 54-56.

[13] J. Chen, W. Zhan and L. Jiang, "A Crowd Evacuation Model Based on 2.5-Dimension Cellular Automaton", Journal of Engineering Graphics, vol. 5, (2009), pp. 170-176.

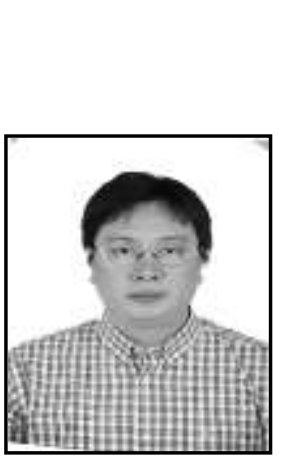

\begin{abstract}
Authors
Fei Fang, he was born in Sichuan Province, China, in 1974. He received the B.S. degree from the Southwest University of China (SWU), Chongqing, in 1997 and the M.S. degree from the Chongqing University of Posts and Tele-communications of China (CQUPT), Chongqing, in 2004. He received the doctor's degree from the School of Communication and Information Engineering, University of Electronic Science and Technology of China (UESTC). His research interests include Wireless Local Area Network, Cognitive radio Wireless network.

Wen-Chun Yu, he was born in Sichuan Province, China, in 1974. He received the B.S. degree from the Sichuan Normal University of China (SICNUU), Chengdu, in 1997 and the M.S. degree from the Southwest Jiao tong University of China (WJITU), Chengdu, in 2009. His research interests include Wireless Local Area Network Large data processing of network and Data Mining.
\end{abstract}

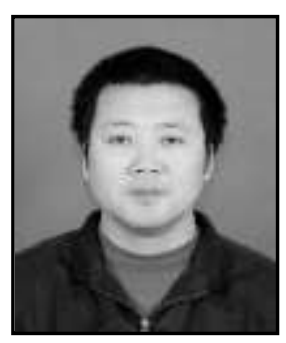

